\title{
Hue distinctiveness overrides category in determining performance in multiple object tracking
}

\author{
Mengdan Sun ${ }^{1} \cdot$ Xuemin Zhang ${ }^{1,2,3} \cdot$ Lingxia Fan $^{1} \cdot$ Luming Hu${ }^{1}$
}

Published online: 13 December 2017

(C) The Psychonomic Society, Inc. 2017

\begin{abstract}
The visual distinctiveness between targets and distractors can significantly facilitate performance in multiple object tracking (MOT), in which color is a feature that has been commonly used. However, the processing of color can be more than "visual." Color is continuous in chromaticity, while it is commonly grouped into discrete categories (e.g., red, green). Evidence from color perception suggested that color categories may have a unique role in visual tasks independent of its chromatic appearance. Previous MOT studies have not examined the effect of chromatic and categorical distinctiveness on tracking separately. The current study aimed to reveal how chromatic (hue) and categorical distinctiveness of color between the targets and distractors affects tracking performance. With four experiments, we showed that tracking performance was largely facilitated by the increasing hue distance between the target set and the distractor set, suggesting that perceptual grouping was formed based on hue distinctiveness to aid tracking. However, we found no color categorical effect, because tracking performance was not significantly different when the targets and distractors were from the same or different categories. It was concluded that the chromatic distinctiveness of color overrides category in determining tracking performance, suggesting a dominant role of perceptual feature in MOT.
\end{abstract}

Keywords Multiple object tracking $\cdot$ Perceptual grouping $\cdot$ Hue $\cdot$ Color category $\cdot$ Categorical perception

The visual system is challenged in a dynamic environment where positions of the objects are changing constantly. How do observers keep track of multiple moving objects at the same time? What differences will it make if these objects possess visual features like color? Multiple object tracking (MOT) is a paradigm that deals with these questions (Pylyshyn \& Storm, 1988). In a classic MOT paradigm, an array of identical objects is presented, among which a subset is

Xuemin Zhang

xmzhang@bnu.edu.cn

1 Beijing Key Laboratory of Applied Experimental Psychology, National Demonstration Center for Experimental Psychology Education (Beijing Normal University), Faculty of Psychology, Beijing Normal University, Beijing 100875, China

2 State Key Laboratory of Cognitive Neuroscience and Learning and IDG/McGovern Institute for Brain Research, Beijing Normal University, Beijing, China

3 Center for Collaboration and Innovation in Brain and Learning Sciences, Beijing Normal University, Beijing, China designated as "targets" in the cueing phase. Participants are required to track these targets while they are moving independently and randomly for a few seconds. While spatiotemporal information is the only source that is used in the classic MOT where objects are identical (Cavanagh \& Alvarez, 2005; Pylyshyn, 1989), recent studies have paid attention to how participants track objects with identity information, such as color (Makovski \& Jiang, 2009a, b), shape (Erlikhman, Keane, Mettler, Horowitz, \& Kellman, 2013; Oksama \& Hyönä, 2008), or face (Ren, Chen, Liu, \& Fu, 2009).

Although earlier findings suggested that the identity of objects is poorly retained in MOT (Bahrami, 2003; Klieger, Horowitz, \& Wolfe, 2004; Pylyshyn, 2006), there has been more and more evidence showing that participants can utilize the identity information to facilitate tracking even when the identity is never tested (Horowitz et al., 2007; Makovski and Jiang, 2009a, b). For instance, Makovski and Jiang (2009b) asked participants to track four targets among a total of eight objects. It was found that tracking all-unique objects (eight objects had eight different colors) was easier for participants than tracking homogeneous objects (eight objects were identical in color). Furthermore, the facilitation of color 
uniqueness in tracking is believed to result from the involvement of working memory, allowing participants to recover lost targets and enhance functional attentional resolution (Makovski \& Jiang, 2009b). However, the uniqueness of objects' features in MOT does not always benefit tracking. It was shown that identity processing had no effect (Botterill, Allen, \& McGeorge, 2011) or even negative effect (Ren et al., 2009) on tracking performance. Liu, Chen, Liu, and Fu (2012) suggested that this full spectrum of behavioral performance of object uniqueness, ranging from facilitation to impairment, depends on how easy it is to process and maintain the identity information. When the target identity is simple and easy to process (e.g., color, one-digit numbers), tracking performance can be improved. When the identity is complex, processing the identity information is resource consuming, which in turn impairs the tracking task (e.g., face, multidigit numbers).

In addition, other researchers manipulated the featural distinctiveness between the target set and distractor set (Erlikhman et al., 2013; Feria, 2012; Howe \& Holcombe, 2012; Makovski \& Jiang, 2009a; Störmer, Li, Heekeren, \& Lindenberger, 2011; Wang, Zhang, Li, \& Lyu, 2016). In the first experiment of Howe and Holcombe's (2012) study, all targets were given a single feature not shared by any of the distractors in the size-distinct condition and the color-distinct condition. The tracking accuracy was much greater in both conditions than in the homogeneous condition, where all objects were identical. What is more, they demonstrated that feature-conjunction targets can increase tracking accuracy as well. Erlikhman et al., (2013) examined whether tracking performance could be increased when the target set was distinct from the distractor set in a variety of features. It was revealed that features - including color, contrast polarity, size, shape, depth, combination (color, shape, size), and interpolationcan be processed to generate target grouping, while orientation cannot. What's more, Erlikhman et al., (2013) categorized these features into four categories by their effects on tracking. The first type - orientation, in their study - is irrelevant to tracking and cannot be processed. The second feature type, voluntary (such as depth), can group the targets when it benefits tracking and can be ignored when grouping is harmful. The third category, automatic nongrouping features (size), can bind targets together to aid tracking but cannot group targets and distractors together. Finally, features such as color are termed as automatic grouping features, which bind objects together automatically, even when the grouping works against the tracking task.

Accordingly, it can be concluded that the featural distinctiveness between the targets and distractors can be used to group targets to aid tracking. The extent of the perceptual grouping effect, however, varies depending on the nature of the particular feature used (Erlikhman et al., 2013). Color, as one of the commonly used features, has been demonstrated to generate target grouping in MOT (Erlikhman et al., 2013;
Howe \& Holcombe, 2012; Makovski \& Jiang, 2009a, b; Störmer et al., 2011). Studies using visual tasks other than MOT have also shown that color is among the strongest attributes influencing image and perceptual organization (Wolfe \& Horowitz, 2004).

However, the processing of color can be more than perceptual. On the one hand, color is associated with the wavelengths of visible light, making it a continuous feature. We perceive colors continuously in terms of hue, lightness, and saturation. A substantial number of studies have found that the chromatic difference in a color space greatly determines the performance in visual tasks (Carter \& Silverstein, 2012; Nagy \& Sanchez, 1990; Olds, Cowan, \& Jolicoeur, 2000). On the other hand, we commonly group colors into discrete categories (e.g., blue, green, yellow). The nature of color categories has been debated extensively (Bird, Berens, Horner, \& Franklin, 2014; Regier \& Kay, 2009; Roberson \& Davidoff, 2000; Roberson, Davies, \& Davidoff, 2000). Some argue that color categories are inbuilt into the visual system (Franklin \& Davies, 2004; Regier, Kay, \& Cook, 2005), while others claim that they are arbitrary linguistic constructs (Roberson et al., 2000). Despite the debate over the origin of color categories, substantial behavioral findings have shown that color categories play a unique role independent of chromaticity in many visual tasks (Bornstein \& Korda, 1984; Boynton, Fargo, Olson, \& Smallman, 1989; Yokoi \& Uchikawa, 2005). Actually, a phenomenon known as categorical perception has shown that discrimination of color stimuli is better across categories than within category (Regier \& Kay, 2009). Yokoi and Uchikawa (2005) showed that search performance is better when the target color is categorically different from the distractor color, compared to when the targets and distractors are categorically identical (the chromatic difference is equal).

This evidence suggested that color categories defined by language may have a unique influence on color-based visual performance, regardless of perceptual chromaticity (Bornstein \& Korda, 1984; Boynton et al., 1989; Yokoi \& Uchikawa, 2005). However, previous MOT studies used considerably different colors as stimuli and have not discriminated between chromaticity and category of color. For instance, Makovski and Jiang (2009a) used eight colors (red, green, blue, yellow, orange, azure, white, and pink) as stimuli in their study to investigate the effect of visual distinctiveness on tracking. The intention of using color as object identity was to dissociate the role of visual distinctiveness from semantic and name distinctiveness, as they stated. However, the colors in their study were not purely visually distinct. Each color was unique in category as well, and easy to label by participants. When color category is involved, the case becomes more complex in MOT. First, MOT is a goal-directed task where participants can voluntarily adopt strategies to aid tracking (Brockhoff \& Huff, 2016; Feria, 2012). Thus, they may use distinct color labels to name objects when the colors are from 
different categories and store them in verbal working memory. In this way, lost targets can be recovered. Second, we might observe color categorical perception in MOT as in other visual tasks; that is, cross-category colors are more distinguishable than within-category colors (Bornstein \& Korda, 1984; Boynton et al., 1989; Yokoi \& Uchikawa, 2005). Participants may group targets based on categorical distinctiveness between targets and distractors to aid tracking. Thus, it is possible that the distinctiveness of chromaticity and category of color may influence tracking in two different ways.

The current study aimed to figure out how chromatic and categorical distinctiveness of color between the targets and distractors affect multiple object tracking performance with four experiments. The purpose of Experiment 1 was to investigate how varying hue distance affects tracking performance when all hues were from the same category (green) in order to exclude the effect of color category. We manipulated hue distance between the target set and distractor set and expected that larger hue distance would boost greater advantage in tracking. In Experiment 2, the category information was introduced in order to explore how both chromatic and categorical differences influence attentive tracking. As in Experiment 1, the hue distance between the targets and distractors varied. At each level of hue distance, the targets and distractors were either within-category or cross-category. If there was a category effect, the tracking performance would be better under the crosscategory condition than the within-category condition when hue distance was equated. The goal of Experiment 3 was identical to that of Experiment 2, but we used different hue samples to test whether the occurrence of category effect will be modulated by the strength of category membership of the hues. In addition, we conducted Experiment 4 to examine the underlying mechanism of the observed effect of the abovementioned three experiments. More specifically, we intended to investigate whether target recovery is the root cause of the effect of color distinctiveness.

\section{Experiment 1}

The purpose of Experiment 1 was to investigate how varying hue distance between the targets and distractors affects tracking performance when the hues were from the same category. We hypothesized that tracking would be facilitated when the targets were distinguished from distractors in hue, compared to when they were all identical.

\section{Method}

\section{Participants}

Sixteen students (12 females; age: $22.8 \pm 2.3$ years) of Beijing Normal University participated in return for monetary compensation. All reported normal or corrected-to-normal sight and normal color perception. Informed consent was obtained before the experimental procedure.

\section{Stimuli and design}

Stimuli were presented on 17-inch CRT monitor, with a resolution of $1024 \times 768$ pixels and refresh rate of $85 \mathrm{~Hz}$. All displays were programmed in MATLAB R2013b (The MathWorks) with Psychophysics Toolbox extensions (Brainard, 1997; Pelli, 1997). Participants were seated approximately $57 \mathrm{~cm}$ away from the monitor; $1 \mathrm{~cm}$ on the screen subtends approximately 1 degree of visual angle $\left(^{\circ}\right)$. The monitor was calibrated to a D65 white point $(x=0.31271, y=$ 0.32902, in CIE 1931 color space) with DataColor Spider5 Colorimeter. The lab environment including the lighting condition, and the position of the monitor was kept unchanged during the whole experiment.

We chose four equally spaced green colors that only varied in hue in CIELAB space $\left(L^{*}=70, a^{*}=0, b^{*}=0\right.$, radius of 58 ; see Fig. 1), the hue angles of which are $130^{\circ}, 140^{\circ}, 150^{\circ}$, and $160^{\circ}$ (see Table 1). The CIELAB uniform color space was derived from threshold color discriminations (Webster \& Kay, 2012). Hence, color space like CIELAB are usually used to control perceptual distance of color stimuli, since color differences across the range of colors are considered roughly equal when the distance of two colors in the space is equaled (Webster \& Kay, 2012; Witzel \& Gegenfurtner, 2013). The RGB values (see Appendix) of these four colors were then generated with the lab2rgb function in MATLAB. These hues were sampled for the moving objects in MOT according to a trial's condition. The moving stimuli were eight disks (diameter $=16$ pixels, visual angel: $0.64^{\circ}$ ) presented on a black

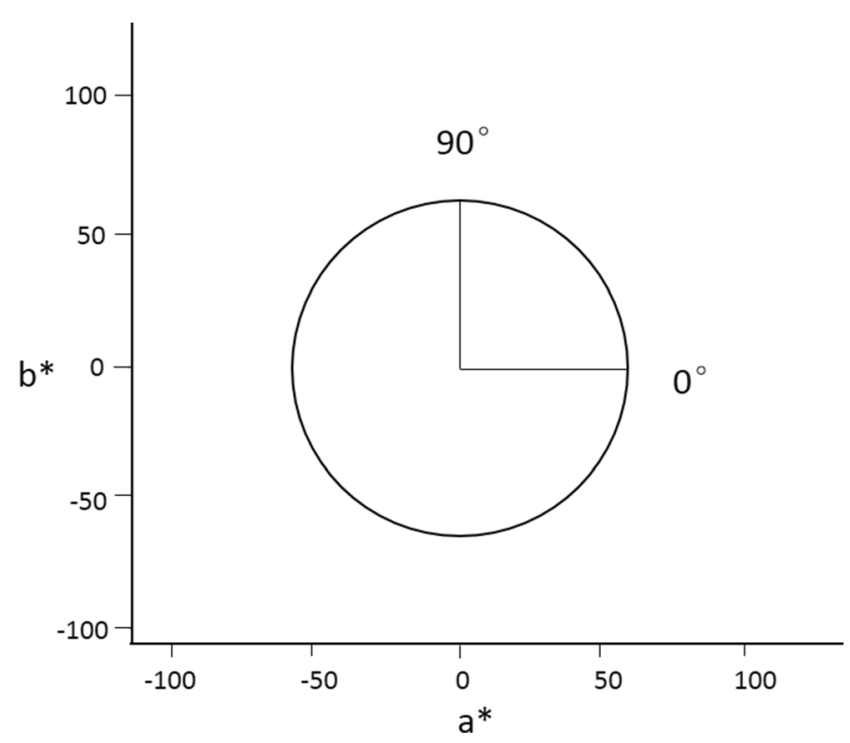

Fig. 1 Hue circle $a^{*}$ and $b^{*}$ coordinates in CIBLAB space 
Table 1 Hues used in MOT of Experiment 1

\begin{tabular}{|c|c|}
\hline Hue Angle & Hue \\
\hline $130^{\circ}$ & \\
\hline $140^{\circ}$ & \\
\hline $150^{\circ}$ & \\
\hline $160^{\circ}$ & \\
\hline
\end{tabular}

Note. These hues are only illustrative and will not be correctly displayed in print or on an uncalibrated monitor

(RGB [0, 0, 0]) background $(800 \times 600$ pixels, visual angle: approximately $32^{\circ} \times 24^{\circ}$ ).

Four conditions differed in hue distance between the target set and distractor set. In the D0 condition, all eight objects were identical in hue, with four types of hue combinations for the targets and distractors: $\left(130^{\circ}, 130^{\circ}\right),\left(140^{\circ}, 140^{\circ}\right)$, $\left(150^{\circ}, 150^{\circ}\right),\left(160^{\circ}, 160^{\circ}\right)$. In the D10 condition, the hue distance between the targets and distractors was $10^{\circ}$ : $\left(130^{\circ}\right.$, $\left.140^{\circ}\right),\left(140^{\circ}, 150^{\circ}\right),\left(150^{\circ}, 160^{\circ}\right),\left(140^{\circ}, 130^{\circ}\right),\left(150^{\circ}, 140^{\circ}\right)$, $\left(160^{\circ}, 150^{\circ}\right)$. In the $\mathrm{D} 20$ condition, the hue distance between the targets and distractors was $20^{\circ}$ : $\left(130^{\circ}, 150^{\circ}\right),\left(140^{\circ}, 160^{\circ}\right)$, $\left(150^{\circ}, 130^{\circ}\right),\left(160^{\circ}, 140^{\circ}\right)$. In the D30 condition, the hue distance between the targets and the distractors was $30^{\circ}$ : $\left(130^{\circ}\right.$, $\left.160^{\circ}\right),\left(160^{\circ}, 130^{\circ}\right)$.

\section{Procedure}

The MOT task consisted of 96 trials, divided randomly and evenly into four conditions. On each trial, eight colored disks were randomly placed inside the white frame. At the beginning of the trial, four of the disks were cued by red boxes for $2 \mathrm{~s}$ to inform the participants that these were the targets to be tracked. Then, all disks started to move independently and randomly at an average speed of 23.04 degree/s. The objects bound off the edge of the frame or repelled one another during the movement. After $6 \mathrm{~s}$, the objects turned white and stopped moving. Participants responded by clicking on four objects and pressed the SPACE key to enter into the next trial.

After the MOT task, participants were asked to complete a color category naming task similar to that in Bae, Olkkonen, Allred, and Flombaum (2015). This task aimed to confirm the intended category membership of the hues used in Experiment 1. On each trial, a disk was present at the center of the screen. The disk was filled with one of the eight colors, four green colors (hue angle: $130^{\circ}, 140^{\circ}, 150^{\circ}, 160^{\circ}$ ) used in the MOT task and four blue colors (hue angle: $210^{\circ}, 220^{\circ}, 230^{\circ}, 240^{\circ}$ ). Participants needed to respond which color term (green or blue) most closely described the color by pressing the key $F$ or $J$. Each participant completed eight trials for each color, a total of 64 trials presented in random order.

\section{Result and discussion}

Figure 2 presented the average tracking performance (proportion of correctly tracked targets) as a function of hue distance. A repeated-measures ANOVA on hue distance produced a significant main effect, $F(3,45)=103.99, p<.001, \eta^{2}=$ 0.87 . Post hoc contrasts using Bonferonni corrections for multiple comparisons revealed that any two conditions were significantly different $(p s<.001)$ except for the D20-D30 pair $(p$ $=1.0$ ). To demonstrate the category membership of four stimulus hues $\left(130^{\circ}, 140^{\circ}, 150^{\circ}, 160^{\circ}\right)$ used in MOT, we presented here the average "green" response rate in the color-naming task (see Table 2). Accordingly, the four hues used in MOT were perceived as green for our participants.

Different from previous studies mixed chromaticity with categories of color, Experiment 1 examined the sole effect of chromatic difference by adopting different hues from the same category, which presented some notable findings. First, the hue difference between the targets and the distractors could significantly affect tracking accuracy even when participants were never asked to attend to the surface property of the moving objects. The magnitude of the difference in tracking accuracy resulting from hue distance was large. The D10 condition (hue distance: $10^{\circ}$ ) was $17 \%$ better than the D0 condition; the D20 condition (hue distance: $20^{\circ}$ ) was $36 \%$ better than the D0 condition. This finding confirmed Erlikhman et al.'s (2013) conclusion that participants can utilize color to group targets. What is more, we carried their work forward by discovering that color-based grouping is largely related to the chromatic

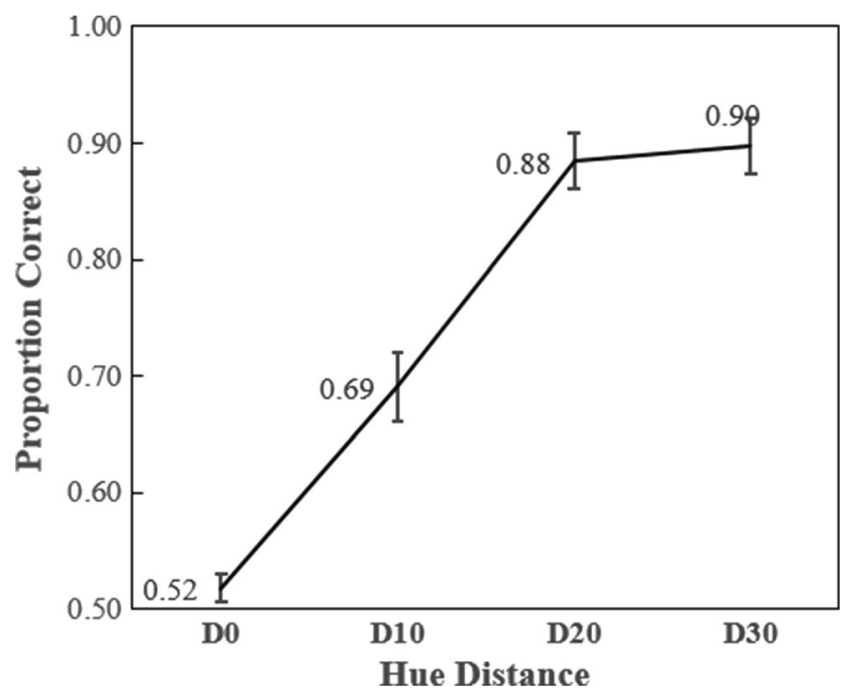

Fig. 2 Tracking performance (proportion correct) as a function of hue distance in Experiment 1 
Table 2 Average "green" response rate in the color category naming task

\begin{tabular}{ll}
\hline Hue angle & Mean $(S D)$ \\
\hline $130^{\circ}$ & $1.00(0.00)$ \\
$140^{\circ}$ & $0.98(0.05)$ \\
$150^{\circ}$ & $0.97(0.07)$ \\
$160^{\circ}$ & $0.95(0.13)$ \\
\hline
\end{tabular}

difference between the targets and the distractors. To further explore how chromaticity and category of color are processed when they concurrently appear in MOT, we conducted Experiment 2, in which colors had distinct features in both hues and categories.

\section{Experiment 2}

The purpose of Experiment 2 was to investigate the effect of distinct categories and hues on tracking performance. The hue distance between the targets and distractors varied. At each level of hue distance, the targets and distractors were either within-category or cross-category. In the within-category condition, the target color and distractor color were from the same category (green). In the cross-category condition, the target color and distractor were from two different categories (green and blue).

\section{Method}

\section{Participants}

Twelve students (12 females; age: $23.3 \pm 2.0$ years) of Beijing Normal University participated in return for monetary compensation. All reported normal or corrected-to-normal sight and normal color perception. Informed consent was obtained before the experimental procedure.

\section{Stimuli and design}

The stimuli in the MOT task for Experiment 2 were identical to those used in Experiment 1, except for the filling colors for the disks. We chose six green colors $\left(155^{\circ}, 160^{\circ}, 170^{\circ}, 175^{\circ}\right.$, $\left.180^{\circ}, 185^{\circ}\right)$ and three blue colors $\left(190^{\circ}, 195^{\circ}, 200^{\circ}\right)$ that only varied in hue in CIELAB space $\left(\mathrm{L}^{*}=70, \mathrm{a}^{*}=0, \mathrm{~b}^{*}=0\right.$, radius of 58; see Fig. 3). A preliminary category naming task was conducted to determine the green-blue boundary. The "blue" response rate in the color-naming task was fitted with a sigmoid function defined as $1 /(1+\exp (-(\mathrm{x}-\alpha) / \beta)$, where $\alpha$ is the threshold (estimated value at which "blue" would be reported half of the time), that is, the green-blue boundary in our study. The results of six participants who did not participate in the formal experiment indicated that the average subjective green-blue boundary was $185.9^{\circ}\left(S D=2.7^{\circ}\right)$.

Seven conditions differed in hue distance and category between the target set and distractor set: D0, Within-D5, CrossD5, Within-D10, Cross-D10, Within-D20, Cross-D20. The hue distance had four levels: $0^{\circ}, 5^{\circ}, 10^{\circ}$, and $20^{\circ}$. In order to test whether participants can form groupings when the hue distance is smaller than 10 degrees, the levels of hue distance were sampled more closely in Experiment 2. Under the D0 condition, the target hue and distractor hue were identical: $\left(160^{\circ}, 160^{\circ}\right),\left(170^{\circ}, 170^{\circ}\right),\left(180^{\circ}, 180^{\circ}\right),\left(185^{\circ}, 185^{\circ}\right)$. Under the conditions of D5, D10, or D20, the target hue and distractor hue are either from the same category (green) in the within-category condition or from different categories (green and blue) in the cross-category condition. The specific hue combinations were as follows. Within-D5: $\left(185^{\circ}, 180^{\circ}\right)$, $\left(180^{\circ}, 185^{\circ}\right)$; Cross-D5: $\left(185^{\circ}, 190^{\circ}\right),\left(190^{\circ}, 185^{\circ}\right)$; WithinD10: $\left(180^{\circ}, 170^{\circ}\right),\left(170^{\circ}, 180^{\circ}\right),\left(185^{\circ}, 175^{\circ}\right),\left(175^{\circ}, 185^{\circ}\right)$; Cross-D10: $\left(180^{\circ}, 190^{\circ}\right),\left(190^{\circ}, 180^{\circ}\right),\left(185^{\circ}, 195^{\circ}\right)$, $\left(195^{\circ}, 185^{\circ}\right)$; Within-D20: $\left(175^{\circ}, 155^{\circ}\right),\left(155^{\circ}, 175^{\circ}\right),\left(180^{\circ}\right.$, $\left.160^{\circ}\right),\left(160^{\circ}, 180^{\circ}\right)$; Cross-D20: $\left(175^{\circ}, 195^{\circ}\right),\left(195^{\circ}, 175^{\circ}\right)$, $\left(180^{\circ}, 200^{\circ}\right),\left(200^{\circ}, 180^{\circ}\right)$.

\section{Procedure}

The MOT task consisted of 168 trials, divided randomly and evenly into seven conditions. The procedure of each trial was identical to that in Experiment 1, except that the objects moved for a random duration between $4 \mathrm{~s}$ and $6 \mathrm{~s}$. After the MOT task, participants also completed a color category naming task, the procedure of which was the same as in Experiment 1, except for the specific colors used. The disk was filled with one of the 12 colors - six green colors and three blue colors used in the MOT task of Experiment 2, and another three blue colors (hue angle: $205^{\circ}, 210^{\circ}, 215^{\circ}$ ). Each participant completed eight trials for each color, a total of 96 trials presented in random order.

\section{Result and discussion}

Analysis of color category naming verified that the average green-blue boundary defined as the point of subjective equality (the estimated value at which "green" would be reported half of the time) lay between $185^{\circ}$ and $190^{\circ}$ (mean $=187.3^{\circ}$; $S D=3.5^{\circ}$ ). Figure 4 presented the average tracking performance as a function of hue distance and category. A repeatedmeasures ANOVA on hue distance (D5, D10, D20) and category (within, cross) produced a significant main effect of hue distance, $F(2,22)=70.48, p<.001, \eta^{2}=0.87$. There was neither a significant main effect of category, $F(1,11)=.01, p=$ $.91, \eta^{2}=.001$, nor a significant interaction effect, $F(2,22)$ $=.37, p=.70, \eta^{2}=.033$. Post hoc contrasts of different hue 


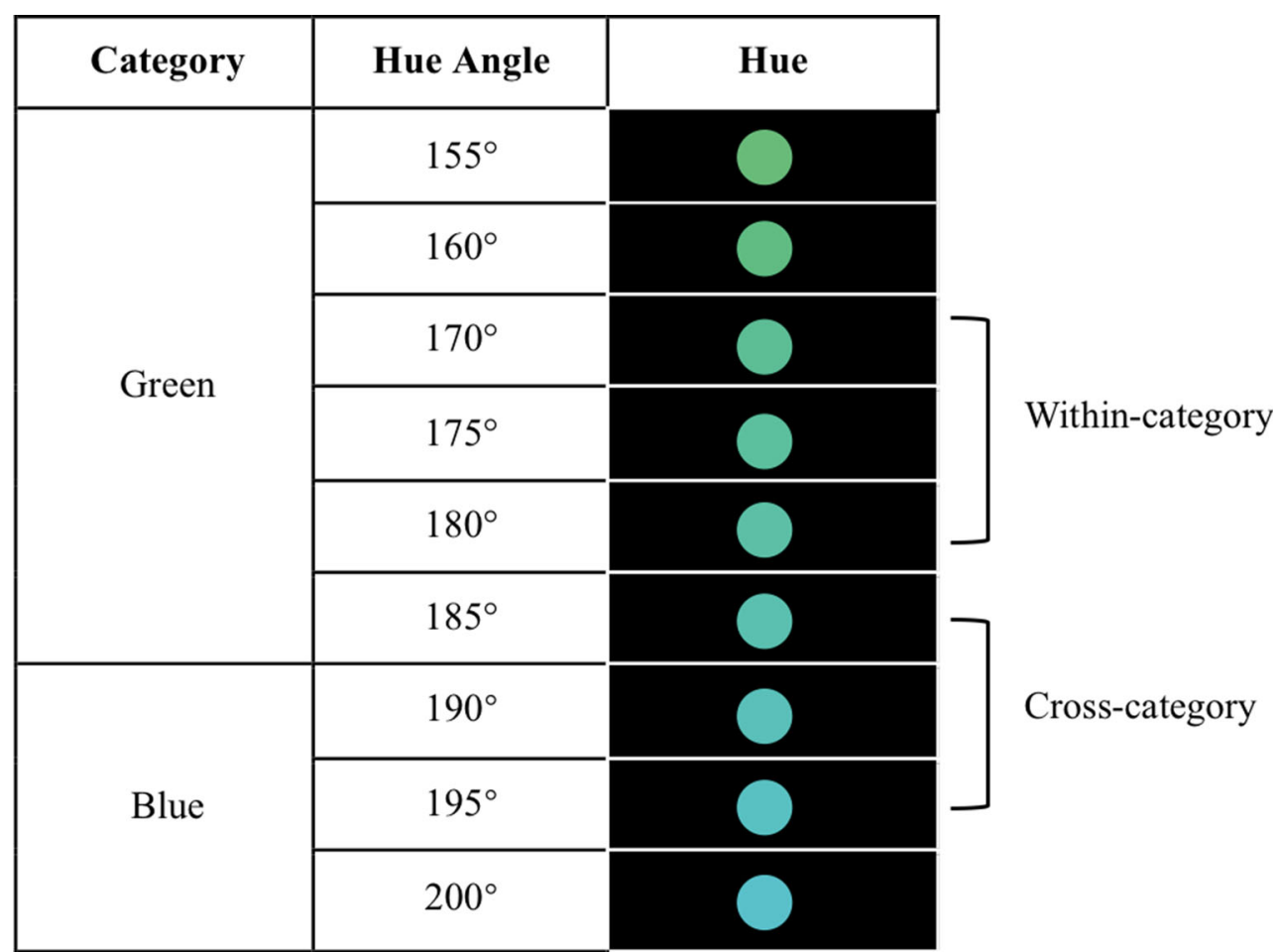

Fig. 3 Hues used in MOT of Experiment 2 and an illustration of within-category and cross-category conditions. Note. These hues are only illustrative and will not be correctly displayed in print or on an uncalibrated monitor. (Color figure online)

distance conditions (D5, D10, D20) using Bonferonni corrections revealed that any two conditions were significantly different (all $p \mathrm{~s}<.001$ ). In addition, a planned $t$ test was conducted on three pairs: D0-D5, D0-D10, and D0-D20, in order to verify the benefit of hue distinctiveness. Significant differences were found in all three pairs: D5-D0, $t(11)=2.23$, $p<.05$, Cohen's $d=0.64$; D10-D0, $t(11)=6.37, p<.001$, Cohen's $d=1.84$; D20-D0, $t(11)=10.85, p<.001$, Cohen's $d$

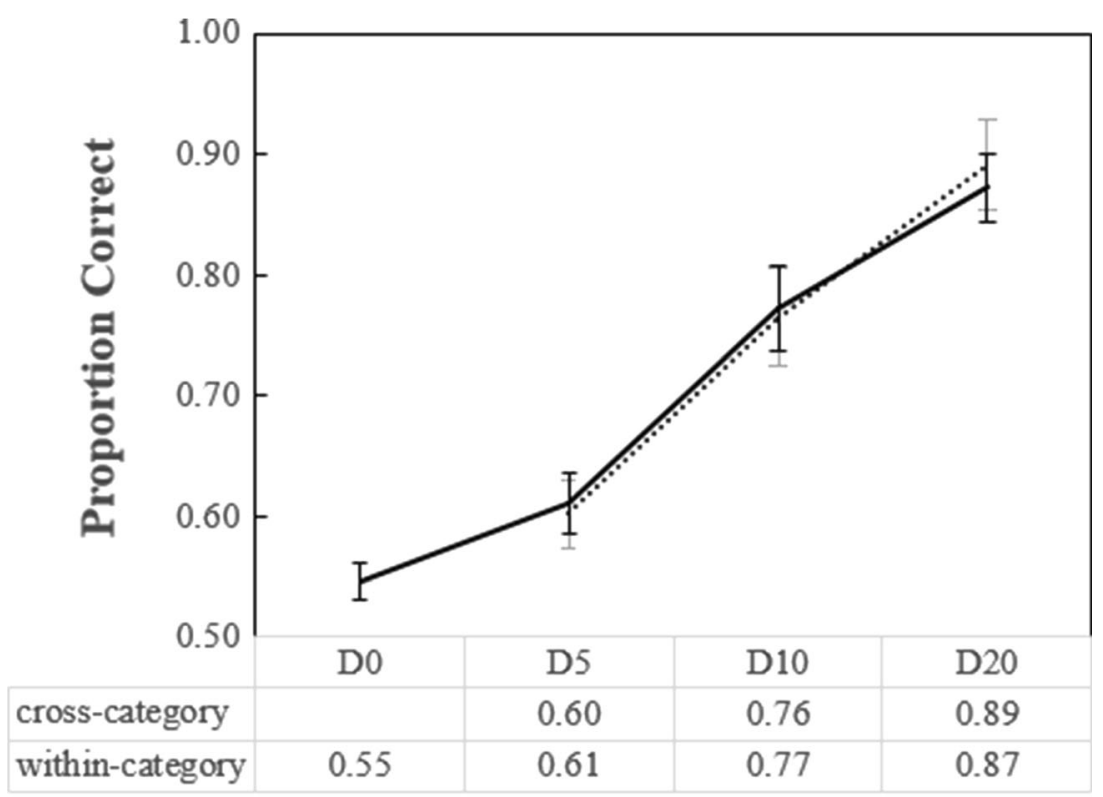

Hue Distance cross-category within-category

Fig. 4 Tracking performance (proportion correct) as a function of hue distance and category in Experiment 2 
$=3.13$. These results suggested that the varying hue distance largely affected tracking performance, while there was no significant difference between within-category and crosscategory colors after controlling the chromatic distance. To examine whether the nonsignificant results of category effect truly supported a null hypothesis, we here also reported Bayes factors $\left.\left(B_{U[0,}, 0.5\right]=0.04\right)$ using an online Bayes calculator (http://www.lifesci.sussex.ac.uk/home/Zoltan_Dienes/ inference/bayes_factor.swf; Dienes, 2014), which showed strong evidence for $\mathrm{H} 0$.

Considering the potential individual differences in color categorization, we conducted another analysis on the effect of category based on an individual green-blue boundary. According to each observer's green-blue boundary, we assigned 20 hue pairs from the D5: $\left(185^{\circ}, 180^{\circ}\right),\left(180^{\circ}\right.$, $\left.185^{\circ}\right),\left(185^{\circ}, 190^{\circ}\right),\left(190^{\circ}, 185^{\circ}\right)$; D10: $\left(180^{\circ}, 170^{\circ}\right),\left(170^{\circ}\right.$, $\left.180^{\circ}\right),\left(185^{\circ}, 175^{\circ}\right),\left(175^{\circ}, 185^{\circ}\right),\left(180^{\circ}, 190^{\circ}\right),\left(190^{\circ}, 180^{\circ}\right)$, $\left(185^{\circ}, 195^{\circ}\right),\left(195^{\circ}, 185^{\circ}\right)$; and D20: $\left(175^{\circ}, 155^{\circ}\right),\left(155^{\circ}\right.$, $\left.175^{\circ}\right),\left(180^{\circ}, 160^{\circ}\right),\left(160^{\circ}, 180^{\circ}\right),\left(175^{\circ}, 195^{\circ}\right),\left(195^{\circ}, 175^{\circ}\right)$, $\left(180^{\circ}, 200^{\circ}\right),\left(200^{\circ}, 180^{\circ}\right)$ conditions into within-category and cross-category conditions. For three observers, all the hue pairs were assigned into the within-category condition under the D5 condition, and thus only nine pairs of valid data were generated. Both of the D10 and D20 conditions included 12 pairs of data after category assignment. All of these pairs were then pooled together, and a paired $t$ test was conducted to compare the tracking performance between within-category and cross-category, which showed no significant differences, $t(32)=1.05, p=.30$, Cohen's $d=0.18$; within-category: 0.76 (0.15); cross-category: $0.78(0.17)$.

Experiment 2 suggested that when the targets distinguished from distractors in both hue and category, the hue distance played a dominant role in affecting tracking, while the categorical distance had no significant influence. Within a slightly different experimental setting, Experiment 2 confirmed what had been found in Experiment 1-that tracking performance increased with the growing perceptual difference between the targets and distractors in MOT. In Experiment 2, the levels of hue distance were sampled more closely. The targets were only 5 degrees different from distractors in hue angle under the D5 condition. Yet we found that the tracking accuracy of the D5 condition was significantly better than the $\mathrm{D} 0$ condition, demonstrating that the visual system is quite sensitive to hue difference in tracking. However, we did not find a main effect of category or interaction effect, suggesting that the category information of colors does not affect the tracking task. To further exclude the possibility that the nonsignificant difference between cross-category and within-category was due to the sampled hues that were quite close to the green-blue boundary and consequently ambiguous in categorization, we conducted Experiment 3, where hues far from the green-blue boundary were included.

\section{Experiment 3}

The purpose of Experiment 3 was to investigate the effect of distinct categories and hues on tracking performance as Experiment 2. However, we adopted hues that were both far and close to the green-blue boundary in order to test whether the null effect of category was caused by the hue sampling. If the distance between the sample hue and the green-blue boundary did influence the effect of category, we should expect an interaction effect between category and hue distance. Here, we reported data from two versions of Experiment 3 that differed in the mask used in the MOT task once objects stopped moving. In Experiment 3a, all colored disks turned white when the movements stopped, as in the above experiments. Experiment $3 \mathrm{~b}$ adopted a colored mask to confirm that the observed effect of color distinctiveness in our study was not due to the lingering afterimage in the response stage.

\section{Experiment 3a}

\section{Method}

\section{Participants}

Eighteen students (15 females; age: $22.7 \pm 2.2$ years) of Beijing Normal University participated in return for monetary compensation. All reported normal or corrected-to-normal sight and normal color perception. Informed consent was obtained before the experimental procedure.

\section{Stimuli and design}

The stimuli in the MOT task for Experiment 3 were identical to those used in Experiment 2, except for the filling colors for the disks. We chose five green colors $\left(140^{\circ}, 165^{\circ}, 170^{\circ}, 175^{\circ}\right.$, $\left.185^{\circ}\right)$ and five blue colors $\left(190^{\circ}, 200^{\circ}, 205^{\circ}, 210^{\circ}, 235^{\circ}\right)$ that only varied in hue in CIELAB space $\left(L^{*}=70, a^{*}=0, b^{*}=0\right.$, radius of 58; see Table 3 ).

Four conditions differed in hue distance and category between the target set and distractor set: Within-D5, Cross-D5, Within-D35, and Cross-D35. The hue distance had two levels: $5^{\circ}$ and $35^{\circ}$. Under the conditions of D5 and D35, the target hue and distractor hue were either from the same category (green or blue) in the within-category condition or from different categories (green and blue, respectively) in the crosscategory condition. The specific hue combinations were as follows: Within-D5: $\left(165^{\circ}, 170^{\circ}\right),\left(170^{\circ}, 165^{\circ}\right),\left(205^{\circ}\right.$, $\left.210^{\circ}\right),\left(210^{\circ}, 205^{\circ}\right)$; Cross-D5: $\left(185^{\circ}, 190^{\circ}\right),\left(190^{\circ}, 185^{\circ}\right)$; Within-D35: $\left(140^{\circ}, 175^{\circ}\right),\left(175^{\circ}, 140^{\circ}\right),\left(200^{\circ}, 235^{\circ}\right),\left(235^{\circ}\right.$, $\left.200^{\circ}\right)$; Cross-D35: $\left(170^{\circ}, 205^{\circ}\right),\left(205^{\circ}, 170^{\circ}\right)$. 
Table 3 Hues used in MOT of Experiment 3

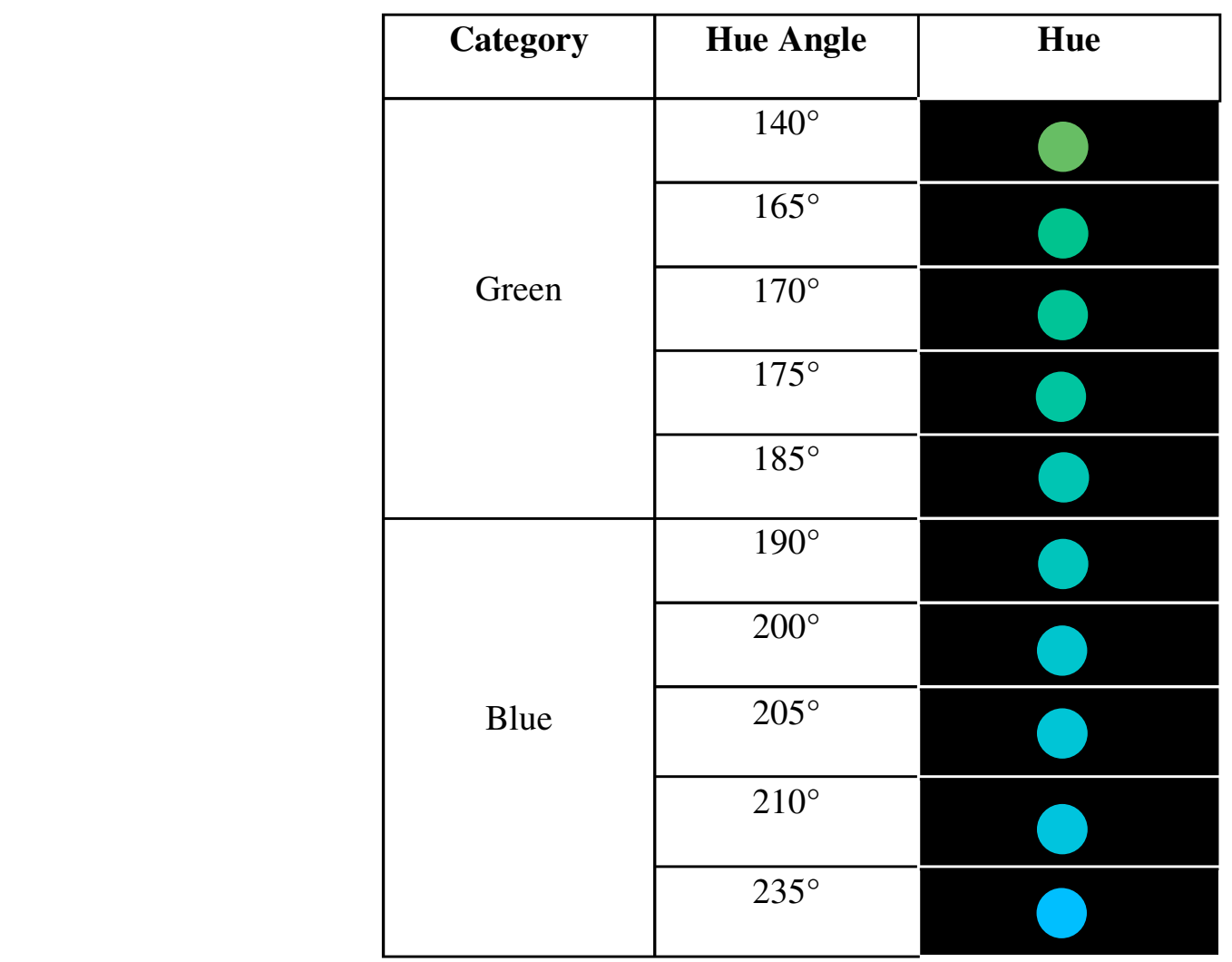

Note. These hues are only illustrative and will not be correctly displayed in print or on an uncalibrated monitor

\section{Procedure}

The MOT task consisted of 96 trials, divided randomly and evenly into four conditions. The procedure of each trial was identical to that in Experiment 2. After the MOT task, participants also completed a color category naming task, the procedure of which was the same as in Experiment 2 except for the specific colors used. The disk was filled with one of the 10 colors used in the MOT task of Experiment 3. Each participant completed eight trials for each color, a total of 80 trials presented in random order.

\section{Results}

Analysis of color category naming verified that the average green-blue boundary, defined as the point of subjective equality (the estimated value at which green would be reported half of the time), lay between $185^{\circ}$ and $190^{\circ}\left(\right.$ mean $\left.=186.8^{\circ} ; S D=4.3^{\circ}\right)$. Figure 5 (left) presented the average tracking performance as a function of hue distance and category in Experiment 3a. A repeated-measures ANOVA on hue distance (D5, D35) and category (within, cross) produced a significant main effect of hue distance, $F(1,17)=1442.05, p<.001, \eta^{2}$ $=0.99$. There was neither a significant main effect of category, $F(1,17)=.29, p=.60, \eta^{2}=.02$, nor a significant interaction effect, $F(1,17)=2.45, p=.14$, $\eta^{2}=.13$. The Bayes analysis of category effect also supported strongly $\mathrm{H} 0$ against $\mathrm{H} 1\left(B_{U[0,0.5]}=0.04\right)$.

In addition, we reexamined the effect of color category on the basis of individual green-blue boundaries following the procedure of Experiment 2. The D5 condition produced seven valid pairs, and the D35 condition produced 18 pairs for analysis. A paired $t$ test showed that the tracking accuracy of within-category was not significantly different from cross-category, $t(24)=.67, p=.51$, Cohen's $d=0.13$; within-category: 0.83 (0.17); cross-category: $0.84(0.18)$.

\section{Experiment 3b}

\section{Method}

\section{Participants}

Eighteen students (17 females; age: $20.6 \pm 1.5$ years) of Beijing Normal University participated in return for monetary compensation. All reported normal or corrected-to-normal sight and normal color perception. Informed consent was obtained before the experimental procedure. 


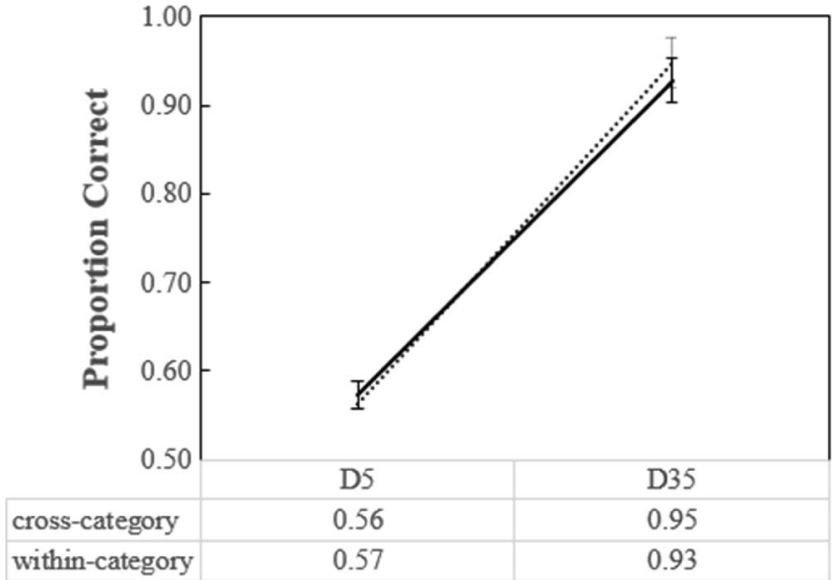

Hue Distance

......... cross-category - within-category

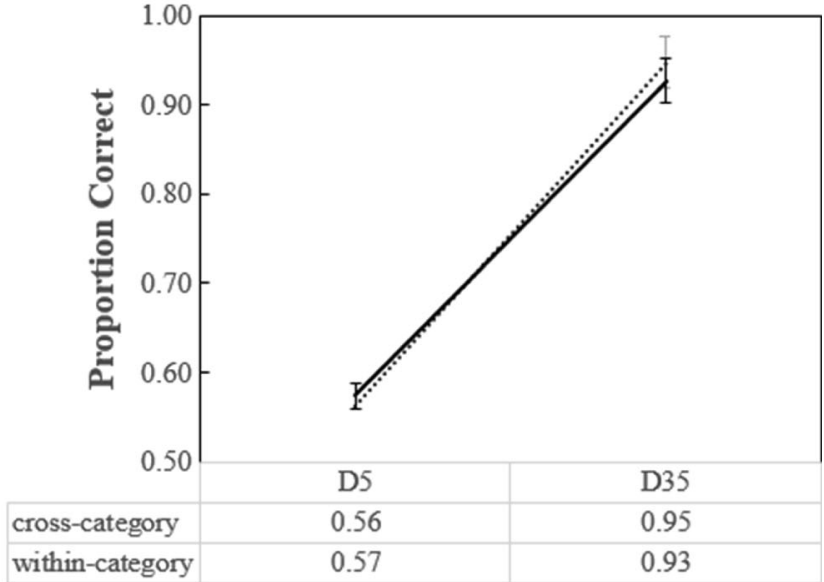

Hue Distance

........ cross-category - within-category

Fig. 5 Tracking performance (proportion correct) as a function of hue distance and category in Experiment 3a (left) and Experiment 3b (right)

\section{Stimuli, design, and procedure}

Experiment $3 \mathrm{~b}$ was identical to Experiment 3a, except for the introduction of the colored mask in the tracking task, similar to Howe and Holcombe's (2012). After the movement stopped on each trial, each disk was covered by a multicolor square mask for $0.25 \mathrm{~s}$. These masks subtended $0.96^{\circ} \times 0.96^{\circ}$ and consisted of three stimuli colors (hue angle: $140^{\circ}, 175^{\circ}$, $235^{\circ}$ ) in a concentric fashion. After the mask was removed, all disks reappeared in white and waited for observers to respond.

\section{Results}

Figure 5 (right) presented the average tracking performance as a function of hue distance and category. A repeated-measures ANOVA on hue distance (D5, D35) and category (within, cross) produced a significant main effect of hue distance, $F(1,17)=1339.81, p<.001, \eta^{2}=0.99$. There was neither a significant main effect of category, $F(1,17)=.04, p=.85, \eta^{2}=$ .002 , nor a significant interaction effect, $F(1,17)=.83, p=$ $.38, \eta^{2}=.05$. The Bayes analysis of category effect also supported strongly $\mathrm{H} 0$ against $\mathrm{H} 1\left(B_{U[0,0.5]}=0.03\right)$.

\section{Discussion}

The results of Experiment 3 suggested that greater hue distance resulted in better tracking performance, while there was no significant difference between within-category and crosscategory colors whenever the cross-category colors were distant or close to the boundary. What is more, Experiment $3 \mathrm{~b}$ further confirmed that the effect of hue distinctiveness was produced in the tracking stage rather than in the response stage. Altogether, with the findings of Experiment 2, it was demonstrated in our study that the chromatic distinctiveness between targets and distractors overrides category distinctiveness in determining tracking performance.

\section{Experiment 4}

Experiments 1-3 have demonstrated that increased chromatic distinctiveness of color between targets and distractors is beneficial to tracking. Yet we wondered whether the chromatic difference improved the tracking per se or just allowed participants to recover targets once they lost them. In order to examine whether target recovery was the only mechanism underlying the hue distinctiveness advantage, we conducted Experiment 4, where participants were asked to track three targets and search for a hidden target during tracking. The participants were told that the hidden target shared the color with the other three targets. The conditions of Experiment 4 were identical to that of Experiment 1, expect for the exclusion of the D0 condition. If the improved MOT performance in Experiment 1 was simply due to target recovery, then the tracking performance in Experiment 4 would be comparable to that of Experiment 1. If it was not, then we expected decreased tracking performance.

\section{Method}

\section{Participants}

Sixteen students ( 13 females; age: $20.6 \pm 2.9$ years) of Beijing Normal University participated in return for monetary compensation. All reported normal or corrected-to-normal sight and normal color perception. Informed consent was obtained before the experimental procedure. 


\section{Stimuli, design, and procedure}

Experiment 4 was identical to Experiment 1, except for the following changes. At the cueing phase of each trial, all eight disks were white, and three targets were designated as targets. After that, all disks started to move and were assigned with hues according to the condition. Participants were instructed at the beginning of the experiment to track three designated targets and search for a hidden target, which shared the same hue of the other three targets, during tracking. Experiment 4 excluded the D0 condition.

\section{Results and discussion}

Figure 6 presents the average tracking performance (proportion of correctly tracked targets) as a function of hue distance under the D10, D20, and D30 conditions in both Experiment 4 (track three targets and search for one target) and Experiment 1 (track four targets). A repeated-measures ANOVA, with hue distance (D10, D20, D30) as the within-subject factor, and task (track three targets and search one target, track four targets) as the between-subject factor produced a significant main effect of hue distance, $F(2,60)=92.23, p<.001, \eta^{2}=0.76$, and task, $F(1,30)=6.76, p<.05, \eta^{2}=0.18$. The interaction between hue distance and task was not significant, $F(2,60)=.05, p=.96, \eta^{2}$ $=0.002$. The main effect of task/experiment suggested that the advantage of hue distinctiveness actually comes from the facilitation of tracking. The target recovery strategy may play a role, but it is not the root cause of the featural distinctiveness effect.

\section{General discussion}

In the experiments presented, we investigated how differences of color, both chromatic and categorical, between the targets and distractors influence multiple object tracking performance. It showed that tracking performance was largely improved by the increasing chromatic distance between the target set and distractor set. However, we found no evidence of color categorical effect-that is, that tracking performance was comparable when the targets and distractors were from the same category or two different categories.

When tracking objects with an identity that is irrelevant to the task (the identity is never tested), the question is no longer whether the identity information is processed during tracking. Rather, researchers are more concerned about what kinds of information can be coded and why (Brockhoff \& Huff, 2016; Erlikhman et al., 2013; Feria, 2012; Liu et al., 2012; ). According to previous studies, both voluntary and involuntary identity processing can be observed in MOT (Erlikhman et al., 2013; Liu et al., 2012; Makovski \& Jiang, 2009a, b). In the current study, we dissociated two levels of information, chromaticity and category of color, and showed the dominant role of distinct chromaticity in affecting tracking performance.

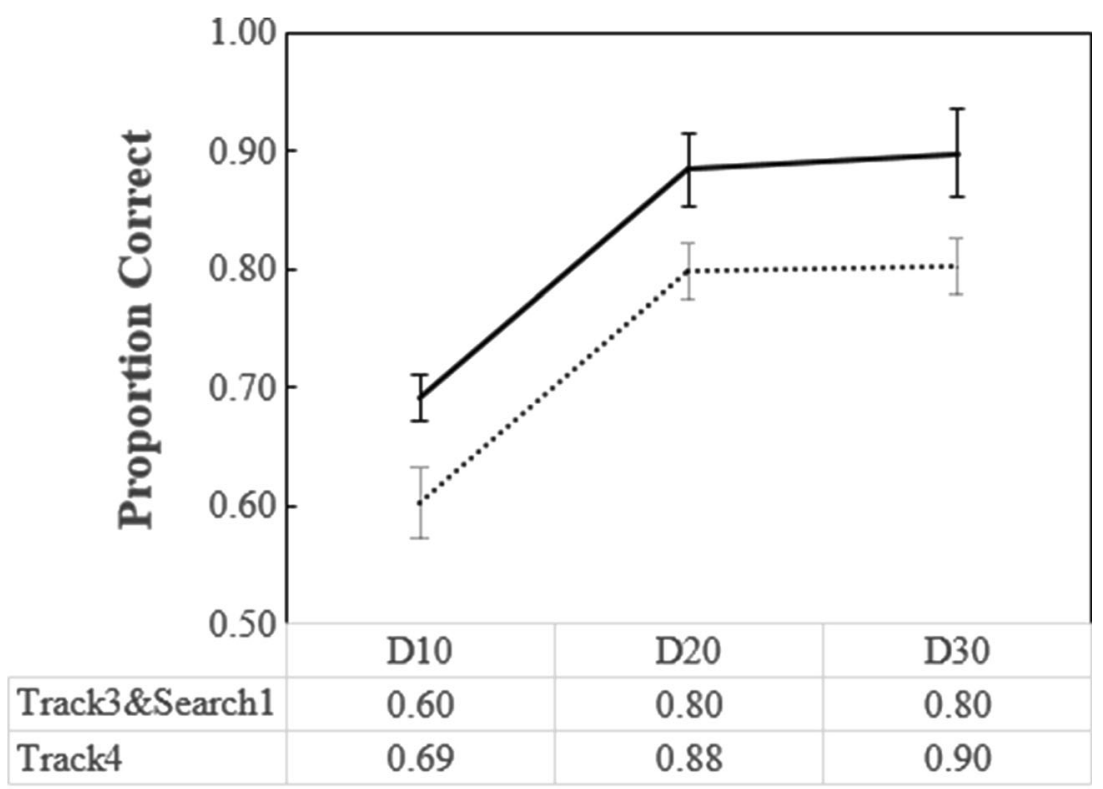

Hue Distance

Fig. 6 Tracking performance (proportion correct) as a function of hue distance and task (track three targets and search one target in Experiment 4; track four targets in Experiment 1) 
According to the evidence from visual search, the coding of color chromaticity occurs at an early stage and guides attention (Duncan, \& Humphreys, 1989; Lindsey et al., 2010). The categories of color, on the other hand, are closely related to verbal naming and language (Webster \& Kay, 2012). We proposed here that the chromatic difference between the targets and distractors invoked involuntary preattentive processing and subsequently facilitated tracking, while the category information of colors was voluntarily not utilized.

The benefit of hue distinctiveness in tracking can probably attribute to perceptual grouping (Yantis, 1992). Yantis (1992) proposed that participants track the targets by grouping them together to form a single virtual object and that attention will then direct toward this coherent but nonrigid object. The grouping effect helps participants to segregate the target set from the distractor set, consequently reducing the error of swapping. When the targets share one single feature that is distinct from the distractor feature, the grouping should become much easier (Erlikhman et al., 2013; Howe \& Holcombe, 2012). When the similarity between the targets and distractors increases, the interference of distractors becomes stronger (Feria, 2012; Störmer et al., 2011). It is the case in our study. Compared to the D0 conditions, tracking accuracy was significantly better in conditions where the targets and the distractors possessed different hues.

What is more, our study suggested that the robustness of perceptual grouping is a continuum and varies as a function of hue distance between the targets and distractors. The grouping advantage increases with the increasing hue distance between target and distractor. Contrary to the notion that distractors are plausibly inhibited during tracking (Bettencourt, Michalka, \& Somer, 2011; Bettencourt \& Somer, 2009; Flombaum, Scholl, \& Pylyshyn, 2008; Pylyshyn, 2006), our findings suggested that the identity information of both targets and distractors, more precisely, their featural relationship, is encoded to generate grouping and guide attention. Studies on visual search and attention have found that search efficiency increases as a function of target-distractor difference and distractor-distractor similarity (Eckstein, 1998; Palmer, Verghese, \& Pavel, 2000; Verghese, 2001). It can be explained by Duncan and Humphreys' (1989) theory that greater target-distractor similarity is harmful to visual search because of the competitive access to visual short-term memory, and the relative weights of targets and distractors is the most important. When generating it to MOT, the virtual object generated by target grouping may become stronger and less breakable by nearby distractors as the perceptual distance between the two sets increases. It allows participants to tolerate a closer distance between a target and distractor (Erlikhman et al., 2013; Feria, 2012).

Furthermore, our studies found that the distinctiveness of category between the targets and distractors had no significant influence on tracking performance. This result suggested two things. First, the verbal labels of colors are not picked up by participants to be stored in working memory for target recovery. Second, color categories do not affect color perception in MOT in a way that makes cross-category colors more discriminable than within-category colors. It is consistent with some evidence from visual search studies suggesting that searching for a target among distractors from a different category is not easier than among distractors sharing the same category (Brown, Lindsey, \& Guckes, 2011; Lindsey et al., 2010). For instance, Brown et al. (2011) asked participants to search for a green target among blue distractors or among a different color of green. No categorical effect was found that the efficiency of visual search for colors relied simply on the lowlevel chromaticity information. Either way, it was demonstrated that color category is negligible in our current settings since it is unable to affect tracking at any level.

However, we cannot fully exclude the possibility that color category could exert a unique role independent of its low-level information when the task is altered. Indeed, previous studies have shown evidence of category-based grouping in MOT (Endress, Korjoukov, \& Bonatti, 2017; Wei, Zhang, Lyu, Hu, \& Li, 2017; Wei, Zhang, Lyu, \& Li, 2016;). Wei et al. (2016; Wei et al., 2017) showed that tracking performance is better when the targets and distractors are from different categories (e.g., animal vs. fruit), relative to when they are from the same category. This finding revealed that, besides the perceptual features, the semantic category of the objects can also be processed to group targets in MOT. They also found that the categorical grouping advantage narrows when the categorical distance decreases. In addition, Endress et al. (2017) presented participants with "mixed" displays of cars and faces or "pure" displays of either cars or faces in a MOT task where all objects were targets. They observed a weak advantage for pure displays, meaning category-based grouping effects could be formed in MOT to some degree. The difference between these studies and our study is that the categories used may be fundamentally different. Categories like animals, cars, and faces are built in to the neural system and have a different representation from one another (e.g., Bonatti, Frot, Zangl, \& Mehler, 2002; Gauthier, Tarr, Anderson, Skudlarski \& Gore, 1999; Kanwisher, McDermott, \& Chun, 1997), while the nature of color categories is still controversial (Bird et al., 2014; Kay \& Kempton, 1984; Regier \& Kay, 2009; Roberson \& Davidoff, 2000; Roberson et al., 2000). This discrepancy could have caused the null effect of color category in our study. What is more, the effect of visual similarity cannot be completely ruled out in those studies, suggesting a category effect (Endress, Korjoukov, \& Bonatti, 2017; Wei et al., 2017, 2016). Endress et al. (2017) speculated that MOT performance might be more sensitive to distinctiveness in perceptual feature than category membership, which is perfectly in line with our findings.

However, we believed that, under some circumstances, color categories will get involved, or even override perceptual features in the MOT task. For example, when the targets are 
each a distinct green hue and distractors are each a distinct blue hue, the category identity is likely to be useful for participants to segregate the targets from the distractors. It was suggested here that categorical perception is an efficient and flexible mechanism of our brain to deal with overloaded perceptual information. With regard to inconsistent findings on categorical effect, Webster and Kay (2012) suggested that interaction between language and color could potentially arise at many different levels, which critically depends on the nature of the task. For instance, if the task has the potential to incorporate verbal categories into the participants' strategies, the effect of categories is likely to occur as a product of verbal coding. In our present study, the perceptual distinctiveness is always accessible and sufficient, and thus it is not surprising for the absence of categorical effect. Future studies can look into this issue in different MOT designs.

Another crucial issue that should not be neglected when investigating the effect of color categories is the impact of individual differences in color categorization (Webster \& Kay, 2012; Witzel \& Gegenfurtner, 2013). In our study, we analyzed the data based on both the average and the individual category boundary to exclude the potential statistical noise, showing the general pattern of noncategorical color coding in MOT. Yet it is possible that the noncategorical effect is not consistent across all individuals. That is, some individuals may show evidence of categorical effect, while others do not. In Webster and Kay's (2012) study, categorical effect occurred in a minority of observers. However, our existing data did not allow us to analyze the effect of color categories at the individual level. Further study could investigate the categorical effect in MOT at the individual level by collecting a greater amount of individual data because both the general patterns and individual differences in categorical effect are informative.

\section{Conclusion}

Our results revealed that the varying distinctiveness in color between the targets and distractors had a significant influence on the tracking performance. Moreover, the tracking accuracy is greatly affected by the chromatic (hue) difference between the two object sets, which may attribute to the effect of perceptual grouping. Besides, we found no evidence of category effect, suggesting that color is not processed categorically in MOT. The perceptual feature of color overrides its category in determining tracking performance.

Acknowledgements This study was supported by the General Program of National Natural Science Foundation of China (31271083) (to XZ) and the Key Program of National Natural Science Foundation of China (61632014) (to XZ).

\section{Appendix}

Table 4 RGB values of the hues used in MOT

\begin{tabular}{llll}
\hline Hue angle $\left({ }^{\circ}\right)$ & $\mathrm{R}$ & $\mathrm{G}$ & $\mathrm{B}$ \\
\hline 130 & 127 & 187 & 86 \\
140 & 103 & 190 & 100 \\
150 & 74 & 193 & 116 \\
155 & 55 & 194 & 124 \\
160 & 23 & 195 & 133 \\
165 & 0 & 195 & 142 \\
170 & 0 & 196 & 151 \\
175 & 0 & 197 & 160 \\
180 & 0 & 197 & 169 \\
185 & 0 & 197 & 179 \\
190 & 0 & 197 & 188 \\
195 & 0 & 197 & 197 \\
200 & 0 & 197 & 206 \\
205 & 0 & 197 & 214 \\
210 & 0 & 196 & 222 \\
235 & 0 & 191 & 255 \\
\hline
\end{tabular}

\section{References}

Bae, G. Y., Olkkonen, M., Allred, S. R., \& Flombaum, J. (2015). Why some colors appear more memorable than others: A model combining categories and particulars in color working memory. Journal of Experimental Psychology: General, 144(4), 744-763. https://doi. org/10.1037/xge0000076

Bahrami, B. (2003). Object property encoding and change blindness in multiple object tracking. Visual Cognition, 10, 949-963.

Bettencourt, K. C., \& Somers, D. C. (2009) Effects of target enhancement and distractor suppression on multiple object tracking capacity. Journal of Vision, 9(7), 9-9

Bettencourt, K. C., Michalka, S. W., \& Somers, D. C. (2011). Shared filtering processes link attentional and visual short-term memory capacity limits. Journal of Vision, 11(10), 22-22

Bird, C. M., Berens, S. C., Horner, A. J., \& Franklin, A. (2014). Categorical encoding of color in the brain. Proceedings of the National Academy of Sciences of the United States of America, 111(12), 4590-4595.

Bonatti, L., Frot, E., Zangl, R., \& Mehler, J. (2002). The human first hypothesis: Identification of conspecifics and individuation of objects in the young infant. Cognitive Psychology, 44(4), 388-426.

Bornstein, M. H., \& Korda, N. O. (1984). Discrimination and matching within and between hues measured by reaction times: Some implications for categorical perception and levels of information processing. Psychological Research, 46(3), 207-222.

Botterill, K., Allen, R., \& McGeorge, P. (2011). Multiple-object tracking: The binding of spatial location and featural identity. Experimental Psychology, 58, 196-200.

Boynton, R. M., Fargo, L., Olson, C. X., \& Smallman, H. S. (1989). Category effects in color memory. Color Research \& Application, 14(5), 229-234.

Brainard, D. H. (1997). The Psychophysics Toolbox. Spatial Vision, 10, 433-436. 
Brockhoff, A., \& Huff, M. (2016). All eyes on relevance: strategic allocation of attention as a result of feature-based task demands in multiple object tracking. Attention, Perception, \& Psychophysics, 78(7), 2090-2109

Brown, A. M., Lindsey, D. T., \& Guckes, K. M. (2011). Color names, color categories, and color-cued visual search: Sometimes, color perception is not categorical. Journal of Vision, 11(12). https://doi. org/10.1167/11.12.2

Carter, R. C., \& Silverstein, L. D. (2012). Perceiving color across scale: Great and small, discrete and continuous. Journal of the Optical Society of America A-Optics Image Science and Vision, 29(7), $1346-1355$

Cavanagh, P., \& Alvarez, G. A. (2005). Tracking multiple targets with multifocal attention. Trends in Cognitive Sciences, 9(7), 349-354.

Dienes, Z. (2014). Using Bayes to get the most out of non-significant results. Frontiers in Psychology, 5(781), 781.

Duncan, J., \& Humphreys, G. W. (1989). Visual search and stimulus similarity. Psychological review, 96(3), 433.

Eckstein, M. P. (1998). The lower visual search efficiency for conjunctions is due to noise and not serial attentional processing. Psychological Science, 9(2), 111-118.

Endress, A. D., Korjoukov, I., \& Bonatti, L. L. (2017) Category-based grouping in working memory and multiple object tracking. Visual Cognition, 1-20

Erlikhman, G., Keane, B. P. Mettler, E., Horowitz, T. S., \& Kellman, P. J. (2013). Automatic feature-based grouping during multiple object tracking. Journal of Experimental Psychology: Human Perception \& Performance, 39,(6), 1625-1637.

Feria, C. (2012). The effects of distractors in multiple object tracking are modulated by the similarity of distractor and target features. Perception, 41, 287-304.

Flombaum, J. I., Scholl, B. J., \& Pylyshyn, Z. W. (2008). Attentional resources in visual tracking through occlusion: The high-beams effect. Cognition, 107(3), 904-931

Franklin, A., \& Davies, I. R. (2004). New evidence for infant colour categories. British Journal of Development Psychology, 22(3), 349-377.

Gauthier, I., Tarr, M. J., Anderson, A. W., Skudlarski, P., \& Gore, J. C. (1999). Activation of the middle fusiform "face area" increases with expertise in recognizing novel objects. Nature Neuroscience, 2(6), $568-573$.

Horowitz, T. S., Klieger, S. B., Fencsik, D. E., Yang, K. K., Alvarez, G. A., \& Wolfe, J. M. (2007). Tracking unique objects. Perception \& Psychophysics, 69, 172-184.

Howe, P. D., \& Holcombe, A. O. (2012). The effect of visual distinctiveness on multiple object tracking performance. Frontiers in Psychology, 27(3), 307. https://doi.org/10.3389/fpsyg.2012.00307

Kanwisher, N., McDermott, J., \& Chun, M. M. (1997). The fusiform face area: A module in human extrastriate cortex specialized for face perception. Journal of Neuroscience, 17(11), 4302-4311.

Kay, P., \& Kempton, W. (1984). What Is the Sapir-Whorf Hypothesis? American Anthropologist, 86(1), 65-79.

Klieger, S. B., Horowitz, T. S., \& Wolfe, J. M. (2004). Is multiple object tracking colorblind? [Abstract]. Journal of Vision, 4(363), 363a.

Lindsey, D. T., Brown, A. M., Reijnen, E., Rich, A. N., Kuzmova, Y., \& Wolfe, J. M. (2010). Color channels, not color appearance or color categories, guide visual search for desaturated color targets. Psychological Science, 21(9), 1208-1214.

Liu, T., Chen, W., Liu, C. H., \& Fu, X. (2012). Benefits and costs of uniqueness in multiple object tracking: The role of object complexity. Vision Research, 66(1), 31-38.

Makovski, T., \& Jiang, Y. V. (2009a). Feature binding in attentive tracking of distinct objects. Visual Cognition, 17(1/2), 180-194.

Makovski, T., \& Jiang, Y. V. (2009b). The role of visual working memory in attentive tracking of unique objects. Journal of Experimental
Psychology: Human Perception and Performance, 35(6), 16871697.

Nagy, A. L., \& Sanchez, R. R. (1990). Critical color differences determined with a visual search task. Journal of the Optical Society of America A-Optics Image Science and Vision, 7(7), 1209-1217.

Oksama, L., \& Hyönä, J. (2008). Dynamic binding of identity and location information: A serial model of multiple identity tracking. Cognitive Psychology, 56(4), 237-283.

Olds, E. S., Cowan, W. B., \& Jolicoeur, P. (2000). The time-course of pop-out search. Vision Research, 40(8), 891-912.

Palmer, J., Verghese, P., \& Pavel, M. (2000). The psychophysics of visual search. Vision Research, 40(10), 1227-1268.

Pelli, D. G. (1997). The VideoToolbox software for visual psychophysics: Transforming numbers into movies. Spatial Vision, 10, 437-442.

Pylyshyn, Z. (1989). The role of location indexes in spatial perception: A sketch of the FINST spatial-index model. Cognition, 32(1), 65-97.

Pylyshyn, Z. W. (2006). Some puzzling findings in multiple object tracking: I. Tracking without keeping track of object identities. Visual Cognition, 11(7), 801-822.

Pylyshyn, Z. W., \& Storm, R. (1988). Tracking multiple independent targets: Evidence for a parallel tracking mechanism. Spatial Vision, 3(3), 179-197.

Regier, T., \& Kay, P. (2009). Language, thought, and color: Whorf was half right. Trends in Cognitive Sciences, 13(10), 439-446.

Regier, T., Kay, P., \& Cook, R. S. (2005). Focal colors are universal after all. Proceedings of the National Academy of Sciences of the United States of America, 102(23), 8386-8391.

Ren, D., Chen, W., Liu, C. H., \& Fu, X. (2009). Identity processing in multiple-face tracking. Journal of Vision, 9(5), 1-15. doi:10.1167/ 9.5.18

Roberson, D., \& Davidoff, J. (2000). The categorical perception of colors and facial expressions: The effect of verbal interference. Memory \& Cognition, 28(6), 977-986.

Roberson, D., Davies, I. R., \& Davidoff, J. (2000). Color categories are not universal: Replications and new evidence from a stone-age culture. Journal of Experimental Psychology: General, 129(3), 369-398.

Störmer, V. S., Li, S. C., Heekeren, H. R., \& Lindenberger, U. (2011). Feature-based interference from unattended visual field during attentional tracking in younger and older adults. Journal of Vision, 11(2), 1-12.

Verghese, P. (2001). Visual search and attention. Neuron, 31(4), 523-535.

Wang, C., Zhang, X., Li, Y., \& Lyu, C. (2016). Additivity of feature-based and symmetry-based grouping effects in multiple object tracking. Frontiers in Psychology, 7, 657. https://doi.org/10.3389/fpsyg. 2016.00657

Webster, M. A., \& Kay, P. (2012). Color categories and color appearance. Cognition, 122(3), 375-392.

Wei, L., Zhang, X., Lyu, C., Hu, S., \& Li, Z. (2017). Brain activation of semantic category-based grouping in multiple identity tracking task. PLOS ONE, 12(5). https://doi.org/10.1371/journal.pone.0177709

Wei, L., Zhang, X., Lyu, C., \& Li, Z. (2016). The categorical distinction between targets and distractors facilitates tracking in multiple identity tracking task. Frontiers in Psychology, 7, 589. https://oi.org/10. 3389/fpsyg.2016.00589

Witzel, C., \& Gegenfurtner, K. R. (2013). Categorical sensitivity to color differences. Journal of Vision, 13(7), 1. https://doi. org/10.1167/13.7.1

Wolfe, J. M., \& Horowitz, T. S. (2004). What attributes guide the deployment of visual attention and how do they do it. Nature Reviews Neuroscience, 5(6), 495-501.

Yantis, S. (1992). Multielement visual tracking: Attention and perceptual organization. Cognitive Psychology, 24, 295-340.

Yokoi, K., \& Uchikawa, K. (2005). Color category influences heterogeneous visual search for color. Journal of the Optical Society of America A-Optics Image Science and Vision, 22(11), 2309-2317. 